\title{
Electric Vehicle Fleet Management using Ant Colony Optimisation
}

\author{
Javier Biera Muriel, Abbas Fotouhi * \\ Advanced Vehicle Engineering Centre, School of Aerospace, Transport and Manufacturing (SATM) \\ Cranfield University, Bedfordshire, MK43 OAL, UK \\ * Corresponding author, a.fotouhi@cranfield.ac.uk; abfotouhi@gmail.com
}

\section{Highlights}

- Specific requirements of an EV fleet management system (FMS) are analysed and a FMS case-study is simulated,

- Ant Colony Optimisation (ACO) technique is used to develop a new ACO-based EV FMS algorithm,

- Comparing to other FMS algorithms such as the nearest-neighbour, ACO's performance is better, however, with additional computational time.

\section{Abstract}

This research is focused on implementation of the Ant Colony Optimisation (ACO) technique to solve an advanced version of the Vehicle Routing Problem (VRP), called fleet management system (FMS). An optimum solution of VRP can bring lots of benefits for the fleet operators as well as contributing to the environment. Nowadays, particular considerations and modifications are needed to be applied in the existing FMS algorithms in response to the rapid growth of electric vehicles (EVs). For example, current FMS algorithms don't consider the limited range of EVs, their charging time or battery degradation. In this study, a new ACO-based FMS algorithm is developed for a fleet of EVs. A simulation platform is built in order to evaluate performance of the proposed FMS algorithm under different simulation case-studies. The simulation results are validated against a well-established method in the literature called Nearest-Neighbour technique. At each case-study, the overall mileage of the fleet is considered as an index to measure the performance of the FMS algorithm.

Keywords: Ant colony optimisation; vehicle routing problem; electric vehicle; fleet management systems. 


\section{Introduction}

The Travelling Salesman Problem (TSP) is defined as a salesman that is required to visit "n" given cities just once, departing from a city or depot and returning to the same point (Lin, 1965). The concern is which of the possible routes is the shortest so the salesman can travel the least. For almost sixty years this problem has been approached from quite a large variety of methods (Ma, Yang, Hou, Tan, \& Liu, 2007; Saji \& Riffi, 2015; Ouaarab, Ahiod, \& Yang, 2013). TSP has real applications in engineering problems as well; a good example is the Vehicle Routing Problem (VRP) that can be considered as form of TSP in which a vehicle is planned to visit a number of cities (Christofides, 1976).

Mathematically, the problem can be defined as a graph $\mathrm{G}=(\mathrm{V}, \mathrm{A})$ where $\mathrm{V}=\{1, \ldots, \mathrm{n}\}$ is a set of vertices that represent cities or customers with the depot located in vertex 1, and $A$ is a set of arcs. Every arc $(i, j) ~ i \neq j$ is associated to an element in the distance symmetrical matrix $\mathrm{C}=\left(\mathrm{c}_{\mathrm{ij}}\right)$ (Laporte, 1992). The aim of the TSP is to minimise the total distance (or cost) covered by the salesman. Let $\mathrm{x}_{\mathrm{ij}}(\mathrm{i} \neq \mathrm{j})$ be a binary variable equal to 1 if and only if the arc $(i, j)$ appears in the optimal solution; therefore, the main objective of the TSP is to find the most optimal route to be followed (Laporte, 1992):

$$
\min \sum_{i \neq j}^{n} c_{i j} * x_{i j}
$$

The way to find the exact solution for the TSP is to calculate all the possible routes and get the best one (depending on the cost function). This can only be implemented if the number of cities or customers is very small. However, as the number of cities increases, the possibilities grow exponentially and the direct search method wouldn't work. So, an advanced optimisation algorithm is needed to be implemented. Along the years, several approaches have been studied in order to solve this problem. One solution is the Nearest Neighbour algorithm that suggests travelling to the closest neighbour from the salesman's current position, which is also named as the "greedy algorithm". Although the process is fairly rapid, a visual analysis shows that the route is far to be optimal: the first distances will tend to be very small, while the last ones are often very large (Boone, Sathyan, \& Cohen, 2015). A useful extension of the TSP is called Multiple Travelling Salesmen Problem (MTSP). An application of MTSP is in VRP where a number of vehicles visit all the given cities (target points). Nature has served as a window display for several 
algorithms to solve the TSP in its different variations and mobile robots path planning (Palmieri, Yang, Rango, \& Marano, 2017; Chen, Kong, Fang, \& Wu, 2011). Genetic Algorithm, Metal Annealing, Neural Networks, Tabu Search and Ant Colony are good examples for this.

A new application of the MTSP which is also considered in this study, is electric vehicle (EV) fleet management system (FMS). There are few studies in the literature focusing on this topic. In a study by Betz, Werner, \& Lienkamp (2016), a mixed fleet of non-electric and electric vehicles was assessed. Then, a new model was developed to investigate the financial and ecological influences of replacing conventional vehicles with EVs to generate a personalized optimal fleet composition according to the number of trips and vehicle specifications. In a study by $\mathrm{Hu}$, Morais, Sousa, \& Lind (2016), a review of the optimization and control methods of intelligent EV fleet charging is discussed and the fleet operator services to other actors in a smart grid are presented. In a study by Chen, Kockelman, \& Hanna (2016), operation of an autonomous EV fleet has been studied by doing simulations. The results have shown that the size of the fleet should be determined based on the charging infrastructure and vehicle range. In another study by Chao \& Xiaohong (2013), differences between a fleet of electric buses and a fleet of diesel buses are assessed. It is shown that such differences need significant changes in vehicle scheduling methods when switching from diesel to electric.

Going through the comparative studies of the theoretical methods that can be considered for VRP, the Ant Colony Optimisation (ACO) technique is one of the most promising techniques in the literature (Tarasewich \& McMullen, 2002). ACO algorithm copies the behaviour of insects, specifically ants due to the clarity of their conduct and big research behind. It is possible to apply ACO in MTSP by using multiple colonies since the multiple colony approach is shown to perform better for larger problems (Bell \& McMullen, 2004). Algorithms like ACO that get the intelligence from individual elements can perform better than the others that work based on a central thinking system (Johnson, 2001). This advantage is quite relevant and applicable to VRP. FMS problem that is studied here, can be considered as a particular type of VRP. Furthermore, ACO can be combined with other algorithms which copy swarm intelligence improving mileage results (Goel and Maini, 2018). 
This study is focused on design and simulation of a FMS algorithm for managing a fleet of commercial EVs. The idea is to utilise an optimisation technique to minimise the overall fleet mileage while visiting a number of target points which are randomly distributed in a surrounded area. Vehicles depart and return to a depot which is located at the centre of the area. Simultaneously, the depot is served as the only charging point (overnight slow charging). This case-study has been introduced in a previous study and a solution was obtained for it using Nearest-Neighbour technique (Fotouhi, et al., 2016). In this study, firstly the Nearest-Neighbour FMS algorithm is improved and secondly, a new FMS algorithm is developed based on ACO technique. Performances of both algorithms are simulated and results are compared. Although, this is not the first time ACO is been used to solve a VRP (Abousleiman \& Rawashdeh, 2014) or the original TSP (Jaradat, 2018). Standing out among the literature, this study is novel in terms of the application of ACO for FMS. In addition, particular requirements of an EV fleet are considered such as EVs' limited travel range and battery degradation minimisation via slow charging overnight.

\section{EV Fleet Management System}

\section{Considerations of an EV Routing Problem}

The main differences, in terms of practicality, between VRP and Electric Vehicle Routing Problem (EVRP) is the necessity of considering the recharging process of the EV battery and its shorter range in comparison to the conventional vehicles (Murakami, 2017). There are different EV charging technologies such as slow charging at depots, fast and super charging between journeys or battery swapping. Each charging technology has its advantages and disadvantages which are out of the framework of this study. The other difference is the EV's range that should be considered when vehicles are dispatched in order to guarantee enough charge to return to depot. In this study, slow charging at depot is considered and the proposed FMS algorithm is able to handle the EV range limitation.

Since charging between journeys is not considered here, the issues due to the charging time are not investigated in terms of time management (assuming slow charging over night at depot) however, this is an important subject which should be addressed in future studies as mentioned in the literature as well (Erdogan \& Miller-Hooks, 2012; Schiffer \& 
Walther, 2017). This is important because fleet operator companies tend to use their fleet at the maximum capacity per day in order to amortize and renew it using the latest technologies. In addition to the charging time, battery degradation and disposal should be also considered. Usually a proper trade-off is needed to make a balance between the charging time and battery degradation. Battery State of Health $(\mathrm{SoH})$ is a quite important factor for today's devices (Barco, Guerra, Muñoz, \& Quijano, 2017).

\section{FMS Case-Study}

The case-study that is considered here includes an optimisation problem in which the overall mileage of a fleet of commercial vehicles is minimised while visiting certain number of target points in a surrounded area. Vehicles depart from and return to a depot which is located at the centre of the area. The depot is served as the only charging point (overnight slow charging). The target points are randomly distributed in a $50 \times 50 \mathrm{~km}^{2}$ area. Different densities are also investigated by changing the number of target points in the same area $(50,200$, and 500 points for low, medium and high density cases) as shown in Fig. 1.

Regarding the slow charging scenario in this case-study, the scheduling plan is prepared for the next day by assuming that target points are known from a day before (an example of this application is the delivery tasks which are performed by commercial fleets). So, each EV starts its journey at fully charged state every day and operates until no more charge is available (by considering the required charge to return to the depot). In terms of the optimisation problem's complexity, this is a simpler case comparing to a dynamic demand scenario like a fleet of taxi where the next target point is unknown. The reason for choosing the simpler scenario in this study, is the time needed for performing the optimisation using ACO (quick real-time optimisation is left for future studies).

Therefore, the role of the FMS algorithm is to allocate the target points to a number of EVs (depending on the number of target points and their distances) in a way to minimise the overall mileage of the fleet. The main constraint here is the range of EV (i.e. identical for all EVs in the fleet). The FMS algorithm would try to use each EV as much as possible however, it should guarantee enough charge for each EV to return to the charging depot 
after completing its task. For the sake of simplicity, no vehicle dynamics, nor topography considerations, nor energy recovery by kinematics or heat is implemented in this study.
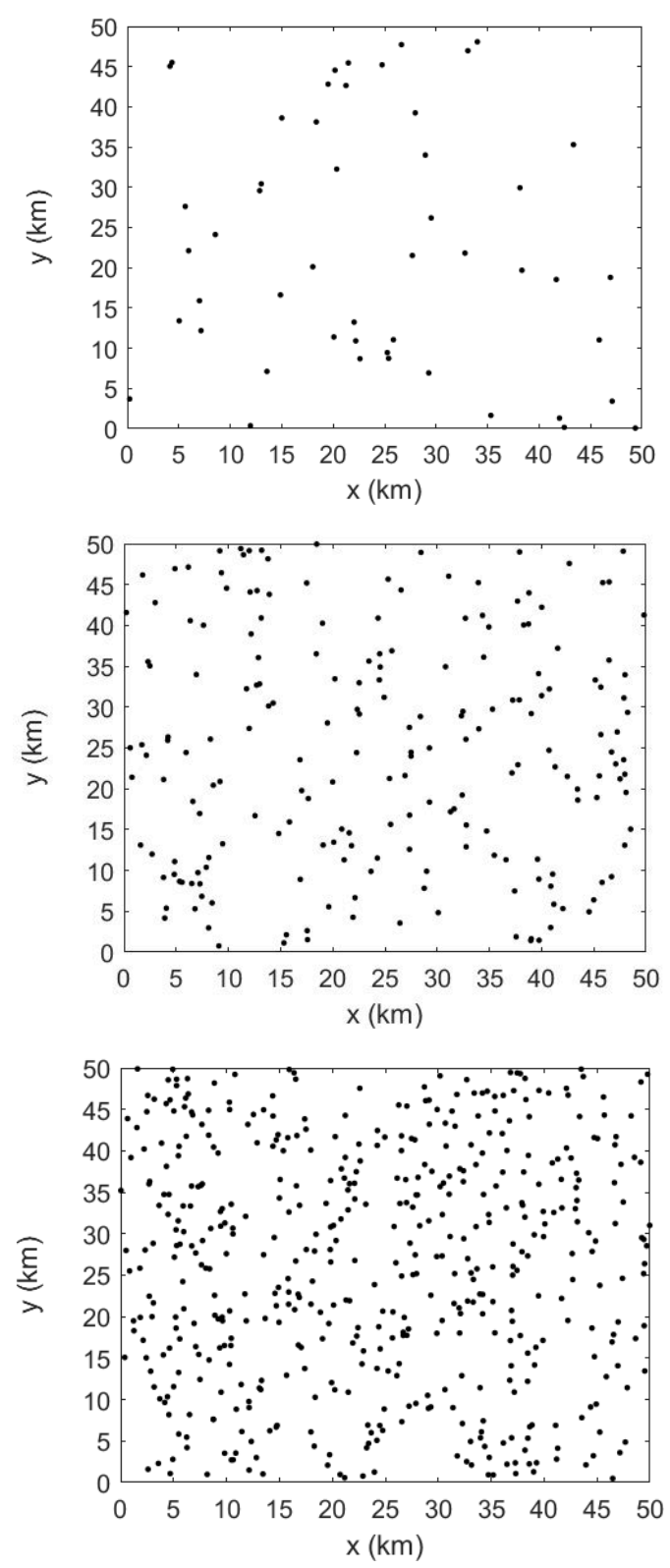

Fig. 1: Random distribution of target points: (a) low density, (b) medium density, and (c) high density

The minimum range of EVs to operate in a $50 \times 50 \mathrm{~km}^{2}$ area is determined based on a previous study by Fotouhi, et al., (2016) in the literature that suggests a range of $125 \mathrm{~km}$ for a similar size area. It should be noted that the main conclusions of this study are not expected to be affected by changing the range of EV. Because the only effect on results when for example we have double range $(250 \mathrm{~km})$, is to dispatch less EVs however, the 
effect is similar to the change of target points' density which is considered in this study. The effectiveness of ACO technique for FMS application is investigated based on the above mentioned case-study however, it is extendable to other case-studies as well.

\section{A New Algorithm for EV FMS using ACO}

In this study, a new EV FMS algorithm is developed using ACO technique. Performance of the proposed algorithm is simulated and analysed for the case-study explained in Section 2.2. As a benchmark to evaluate the simulation results, the Nearest-Neighbour FMS algorithm is also implemented and the results are compared. The two techniques are fundamentally different; while the Nearest Neighbour-based FMS algorithm always tends to travel to the closest point from the current position, the ACO-based FMS algorithm considers a wider range of different options to travel.

A basic version of the Nearest Neighbour-based FMS algorithm has been developed in the literature by Fotouhi, et al., (2016). However, in this study, improved versions of the Nearest Neighbour technique are proposed and used that are called 2 Point Optimisation (2-Opt) and 3 Point Optimisations (3-Opt) Nearest Neighbour algorithm. In both algorithms, the original signal that is obtained from Nearest Neighbour is rechecked for possible improvements by selecting consecutive points and evaluating all possible scenarios to pass them to find the shortest one. In 2-Opt, each two consecutive points are rechecked whereas in 3-Opt, same procedure is performed for each three consecutive points. These techniques are helpful when a route line crosses itself. This is a very common situation in Nearest Neighbour, especially in high city density scenarios. Computation time increases, although the reduction in overall mileage over additional time is worth the effort (Lin, 1965).

The Nearest Neighbour-based FMS algorithm is used in this study just as a benchmark to evaluate ACO-based FMS algorithm.

\section{Introduction to $\mathrm{ACO}$}

While an ant is walking it leaves pheromones along the path, the artificial pheromones in the ACO algorithm represent the desirability of a path. The more ants decide to take that path the more pheromones will be deposited and then the route is identified as a desired 
route (Narasimha \& Kumar, 2011). The more ants are over the graph, the more information will be available and consequently a better result might be achievable. Nevertheless, the technique requires additional computational effort. There are three clues to understand the formation of optimum paths with ACO: "(i) the preference for paths with a high pheromone level, (ii) the higher rate of growth of the amount of pheromone on shorter paths, and (iii) the trail mediated communication among ants" (Dorigo \& Gambardella, 1997). In addition, 'artificial' ants have the memory of the visited points so they can perform the whole TSP (Bell \& McMullen, 2004). In such a scenario, ants are guided along the possible routes between cities. In these kind of self-learning algorithms the solution is not unique and might change at every attempt.

The ACO equations are designed to give a percentage of randomness in finding the solutions that can help to avoid 'local minima' solutions. In general, it is very difficult to find the 'global minima' for TSP especially when the number of points increases. So, here we are talking about semi-optimum solutions and there is no mathematical prove that the result of ACO is the best. As mentioned before, the nearest-neighbour algorithm is used as a benchmark in all the simulation cases in order to have a better judge about the ACO performance.

Parameter initialisation is something important that the ACO's performance relies on it. Indeed, using the most suitable parameters is a matter of fact to get the best results. The ACO initialisation process contains determination of general parameters such as the number of iterations and the number of ants (which affect the simulation time and the algorithm convergence) and also more specific parameters (listed in Table 1) which affect the algorithm's performance in terms of ant behaviour. Table 1 gives a brief explanation about four main parameters in ACO. In this study, the ACO parameters are tuned by repeating the simulations using different set of parameters and considering the average performance. 
Table 1: ACO Parameter Explanation

Parameter

Evaporation ratio, $\rho$ (rho)

Pheromone exponential weight, $\alpha$ (alpha)
This parameter defines the percentage of pheromones that remain after every iteration, $\rho$ changes between 0 and 1
This parameter weights the amount of initial and new pheromones when pheromone concentration is updated, $\alpha$ changes between 0 and 1
Heuristic exponential weight, $\beta$ (beta)
This parameter weights the distance and pheromone trail, $\beta$ changes between 0 and $\infty$

Next customer selection parameter, $\mathrm{Q}_{0}$ customer selection, $\mathrm{Q}_{0}$ changes between 0 and 1

\section{FMS Algorithm using ACO}

In this study, a novel FMS algorithm is developed for a fleet of EVs to operate based on the case-study presented in Section 2.2. ACO technique is used as the main part of the proposed FMS algorithm. Literature has covered ACO to solve the EV routing problem, even going further in complexity than this paper's approach, such as Zhang et al. (2018). However, this research brings to front page the fleet behaviour using ACO, which is not considered in literature. The algorithm is developed and simulated using MATLAB software. As a starting point of the code generation process, a basic ACO code is used from the literature (Heris, 2015). Essential changes have been applied to the original ACO algorithm in order to make it applicable for EV FMS. As a result, a new algorithm has been developed as shown in Fig. 2 in form of a flow chart. One of the main changes applied in the baseline ACO code to propose an ACO-based FMS algorithm is the inclusion of several EVs. While the baseline algorithm performs the whole problem with one agent (i.e. a vehicle in this case), the proposed FMS algorithm is able to dispatch several EVs due to their limited range. Furthermore, the proposed algorithm has different parts including initialisation, iterations, ants' state updating (inside iterations) and ants' pheromone updating. There are five main functions in the algorithm: 1) determination of the first point to start, 2) investigation of possible points to travel next, 3) selection of the next point to travel, 4) pheromone updating, and 5) pheromone evaporation. The number of iterations and the number of ants are initialised at the beginning. 
Determination of the first point to travel can be performed by a random function, so any city can be chosen. This piece of randomness in the FMS algorithm can lead to some gain in the performance since it contributes to algorithm's exploration. However, a previous study in the literature by Fotouhi, et al. (2016) demonstrates that the initial point in such a FMS case-study is quite important. Although, the results are shown for Nearest Neighbour-based FMS algorithm, this can be extended to ACO algorithm as well. To investigate this further, both the random (normal) initialisation and 'best point' initialisation approaches are simulated in Section 4. The 'best point' initialisation approach comes from the literature (Fotouhi, et al., 2016) where all the existing points are tested as the initial point at the beginning and the one that gives the best result is selected. Previous research shows an impressive improvement with the implementation of the best initial condition for Nearest Neighbour technique. In this study, firstly the best initial condition is determined using the Nearest Neighbour technique and then ACO starts from that point instead of a random point. The process of 'best initialisation' can be performed only for the first EV or it can be repeated every time when a new EV is dispatched (i.e. for all EVs at the beginning of their journeys). The results of all initialisation techniques are investigated in Section 4.

After travelling to the first point, all other points are considered as a possible candidate to be the second point. However, some of them might not be feasible because of the EV range limitation. In addition to the unfeasible points in term of EV range, the visited points should be also eliminated from the list of possible next points. This will be the input for the next customer function that selects the next point by comparison of two variables $\mathrm{Q}_{0}$ (parameter, $0 \leq \mathrm{Q}_{0} \leq 1$ ) and $\mathrm{q}$ (random variable with uniform probability, [0,1]) as follows (Dorigo \& Gambardella, 1997; Bell \& McMullen, 2004):

$$
\text { if } Q_{0} \leq q \quad j=\underset{\text { otherwise } S}{\operatorname{argmax}}\left\{\left(\tau_{\text {iu }}\right)\left(\eta_{\text {iu }}\right)^{\beta}\right\} \quad u \notin M_{k}
$$

where $i$ is the current position and $u$ is the next one, $\tau$ is the pheromone concentration, $\eta$ is a heuristic function taken from the inverse of the distance between cities $i$ and $u, \beta$ is a parameter that weighs the distance and pheromone trail $(\beta>0), M_{k}$ is a vector that contains all visited cities. The parameter $s$ is a random variable based on the probability 
distribution $P$, it will favour short routes with high level of pheromones (Bell $\&$ McMullen, 2004):

$$
P_{i s}=\frac{\left(\tau_{i s}\right)\left(\mu_{i s}\right)^{\beta}}{\sum_{u \in M_{k}}\left(\tau_{i u}\right)\left(\mu_{i u}\right)^{\beta}} \quad \begin{gathered}
s \notin M_{k} \\
\text { otherwise } 0
\end{gathered}
$$

Pheromone updating for TSP has been approached from the point of view that every ant affects the pheromone concentration as it happens in real life. However, previous research in EVRP models show that better behaviour is observed when pheromones are updated if and only if a better solution is reached, hence, not all ants will contribute to pheromone updating (Abousleiman \& Rawashdeh, 2014). The pheromone updating equation will select a percentage of the initial and new pheromones (Bell \& McMullen, 2004):

$$
\tau_{i j}=(1-\alpha) \tau_{i j}+(\alpha) \tau_{0}
$$

where $\alpha$ is a parameter that weighs the amount of initial and new pheromone that will remain $(\alpha(0,1)), i$ and $j$ are the current and next position, $\tau_{0}$ is the initial pheromone concentration ruled by the following formula (Heris, 2015):

$$
\tau_{o}=\frac{10 * Q_{0}}{\text { number of cities } * \text { mean (distance matrix })}
$$

Pheromone evaporation takes place before the start of a new iteration (Heris, 2015):

$$
\tau_{i j}=(1-\rho) \tau_{i j}
$$

Where $\rho$ is the evaporation ratio.

Referring to Fig. 2, inside every iteration loop, there is another loop regarding the number of ants. At the end of each stage, a cost function is calculated (i.e. the overall mileage of the fleet) and the new solution is saved if it outperforms the previous best solution. The same check is done when updating the pheromone concentration via Equation 4. Before going to the next iteration, the best solution is updated if necessary so the algorithm can keep on improving. Additionally, parameters will ensure proper development of the solutions as explained before. The solution might not improve after a certain number of iterations. This is due to a stabilisation in pheromone concentration over the best solution or sometimes an excessive pheromone evaporation. 


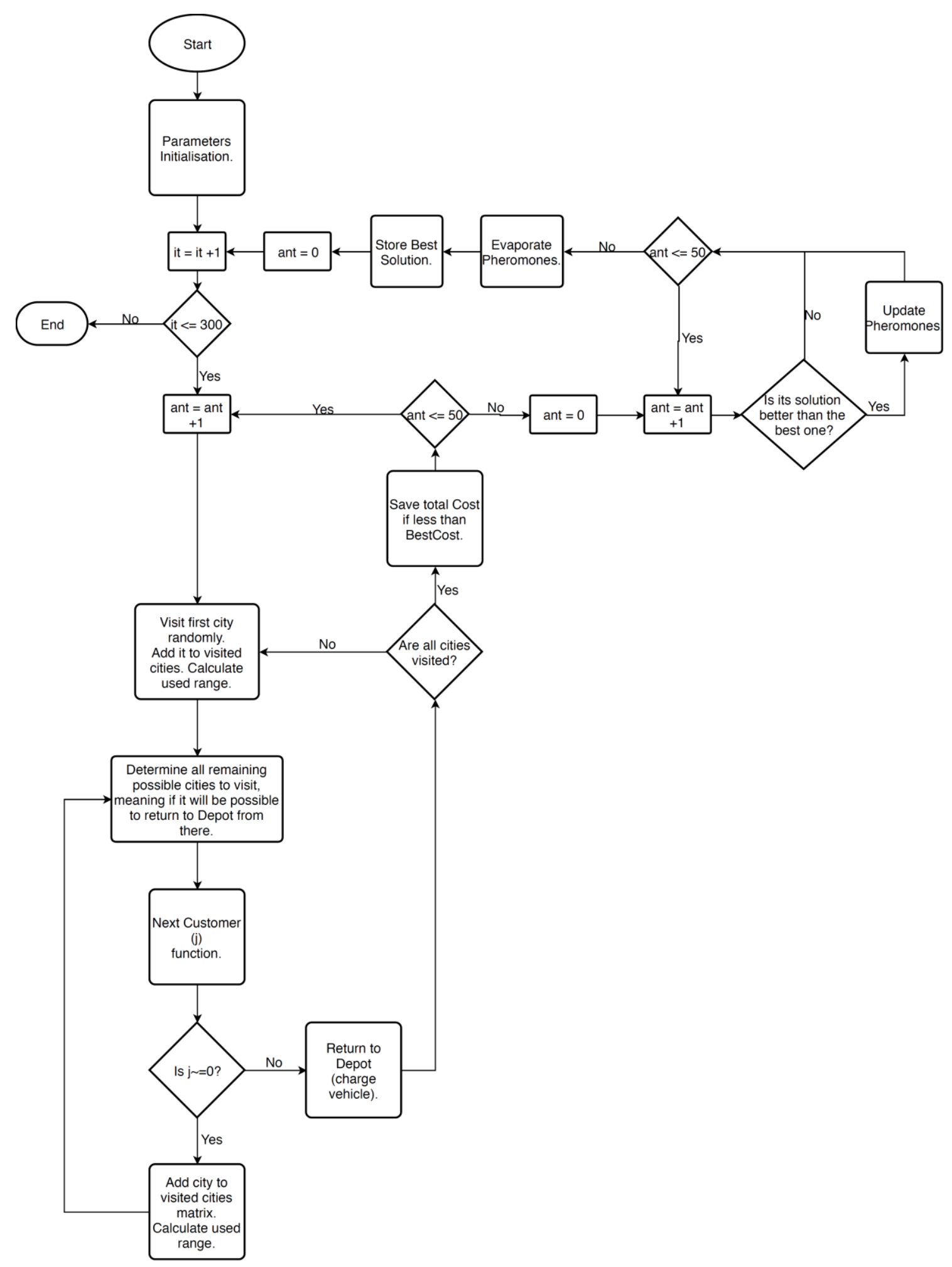

Fig. 2: ACO-based FMS algorithm's flow chart 


\section{FMS Simulation and Results Analysis}

In this section, the proposed ACO-based FMS algorithm is simulated for the case-study explained in Section 2.2. For each simulation case, the overall mileage of the fleet is considered as the 'cost function' to evaluate performance of the FMS algorithm. Indeed, a better performance is defined in a way to achieve less total mileage while reaching all the target points (i.e. the constraint of the optimisation problem).

In order to have a comprehensive study of the ACO-based FMS algorithm, adjustable parameters of the ACO algorithm are changed and performance of the fleet is investigated in each case separately. In addition, the effect of initial condition on FMS performance is investigated.

\section{FMS simulation using different values of ACO parameters}

In this section, the effects of ACO parameters on the FMS performance are investigated including the number of ants, the number of iterations, pheromone evaporation rate, pheromone concentration, weighting of pheromone trail with distance, and next city selection process. All the simulation cases are performed for the medium density (as defined earlier in this article) to keep consistency.

\section{Number of ants}

To investigate the impact of the number of ants on FMS performance, the FMS casestudy is simulated using different numbers of ants. Table 2 contains average and the best results of FMS algorithm in term of the total fleet mileage using different number of ants varying from 20 to 100 . The results demonstrate that increasing the number of ants doesn't necessarily improve the FMS performance. This result can be explained in a way that having more ants implies more pheromone information, hence less accuracy from a certain value. On the other hand, having less ants would lead to lack of pheromone concentration. So, an optimum number of ants can be obtained for each application that is 50 ants in this case based on Table 2. 
Table 2: the effect of number of ants on overall fleet mileage

\begin{tabular}{ccc}
\hline number of ants & $\begin{array}{c}\text { average result } \\
(\mathbf{k m})\end{array}$ & $\begin{array}{c}\text { the best result } \\
(\mathbf{k m})\end{array}$ \\
\hline 20 & 723.29 & 690.40 \\
50 & 707.25 & 682.22 \\
75 & 714.02 & 701.37 \\
100 & 712.86 & 707.14 \\
\hline
\end{tabular}

\section{Number of iterations}

According to the theory of ACO, by running the algorithm longer, the better results might be achieved but with additional computational time. So, a proper trade-off is needed to determine the optimum number of iterations based on the required level of accuracy in each application. Table 3 contains average and the best results of FMS algorithm in terms of the total fleet mileage using different number of iterations. As stated in the table, the simulation is performed by considering 200, 500, and 1000 iterations. It should be noted that each simulation case is repeated a number of times to avoid inaccuracy due to the randomness that exists in ACO algorithm. Both average and best result are presented for each case in Table 3. The results demonstrate that more iterations improve the results however, the rate of improvement decreases after a certain number of iterations. At that point, the computational time increases without enough benefit in terms of FMS performance that means a proper trade-off is needed between them. According to the results presented in Table 3, the number of iterations is set at 500 .

\begin{tabular}{|c|c|c|}
\hline $\begin{array}{l}\text { number of } \\
\text { iterations }\end{array}$ & $\begin{array}{c}\text { average result } \\
(\mathbf{k m})\end{array}$ & $\begin{array}{c}\text { the best result } \\
(\mathrm{km})\end{array}$ \\
\hline 200 & 709.35 & 689.19 \\
\hline 500 & 702.44 & 680.47 \\
\hline 1000 & 702.67 & 692.99 \\
\hline
\end{tabular}

\section{Pheromone concentration, alpha ( $\alpha)$}

This parameter weights the pheromone concentration that will be deposited if a solution better than the best one is found. According to the ACO formulation, a percentage of the initial pheromone will remain and some new pheromone is added into the system. Short paths will tend to accumulate pheromones quicker than non-desired routes so, the pheromone concentration reflects path desirability. Fig. 3 shows the effect of pheromone 
concentration factor on overall fleet mileage. According to the results, the optimum value of this parameter is selected to be 0.75 in this case-study.

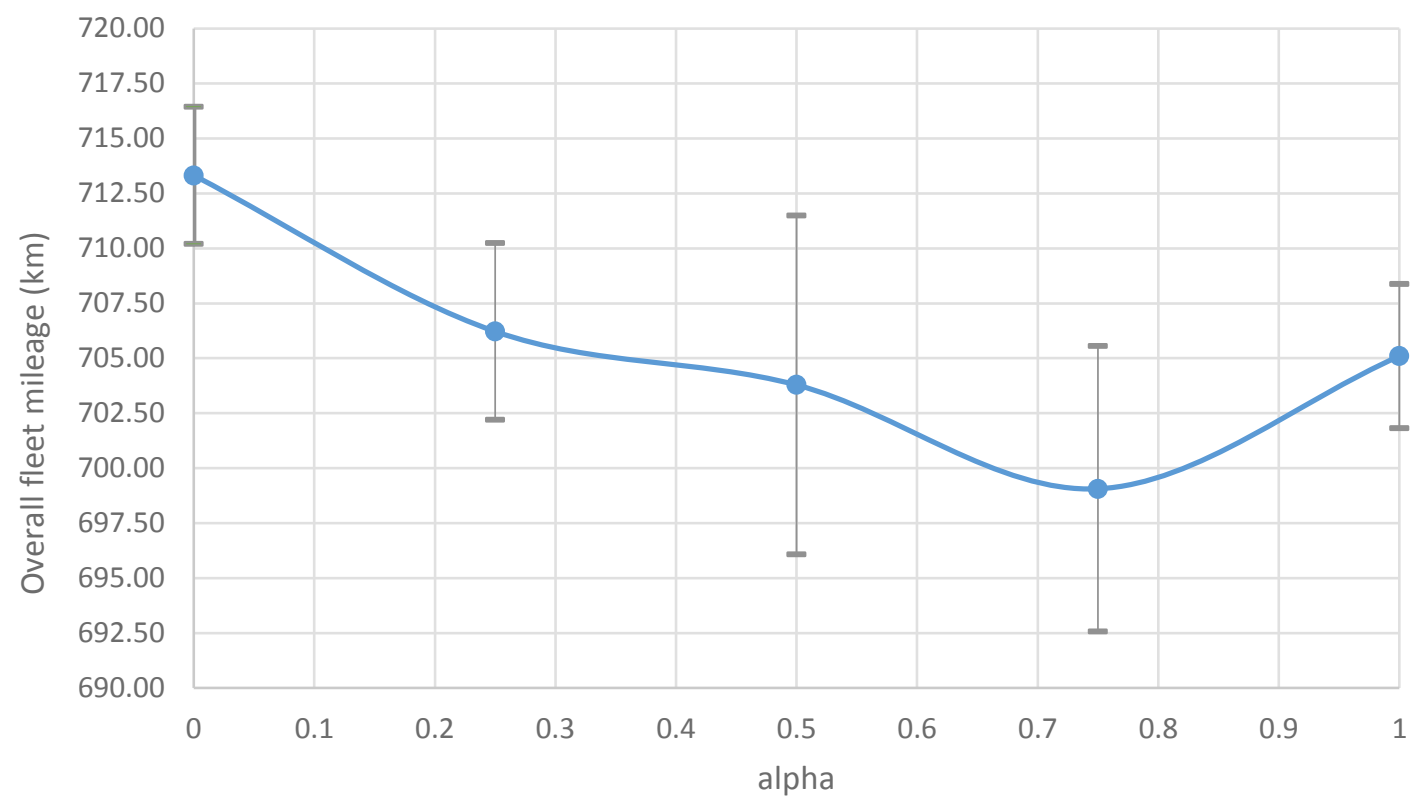

Fig. 3: The effect of pheromone concentration factor (alpha) on overall fleet mileage

\section{Pheromone evaporation, rho ( $\rho)$}

This parameter implements a useful form of forgetting in the ACO algorithm. This is useful for exploration of new areas in the search space. Fig. 4 shows the effect of pheromone evaporation factor (rho) on overall fleet mileage including standard deviations, average and best values. Based on the results in Fig. 4, the value of rho around 0.3 gives the best outcome for this case-study.

For more details about pheromone updating mechanism in ACO algorithm refer to Zhang \& Zhang (2018). 


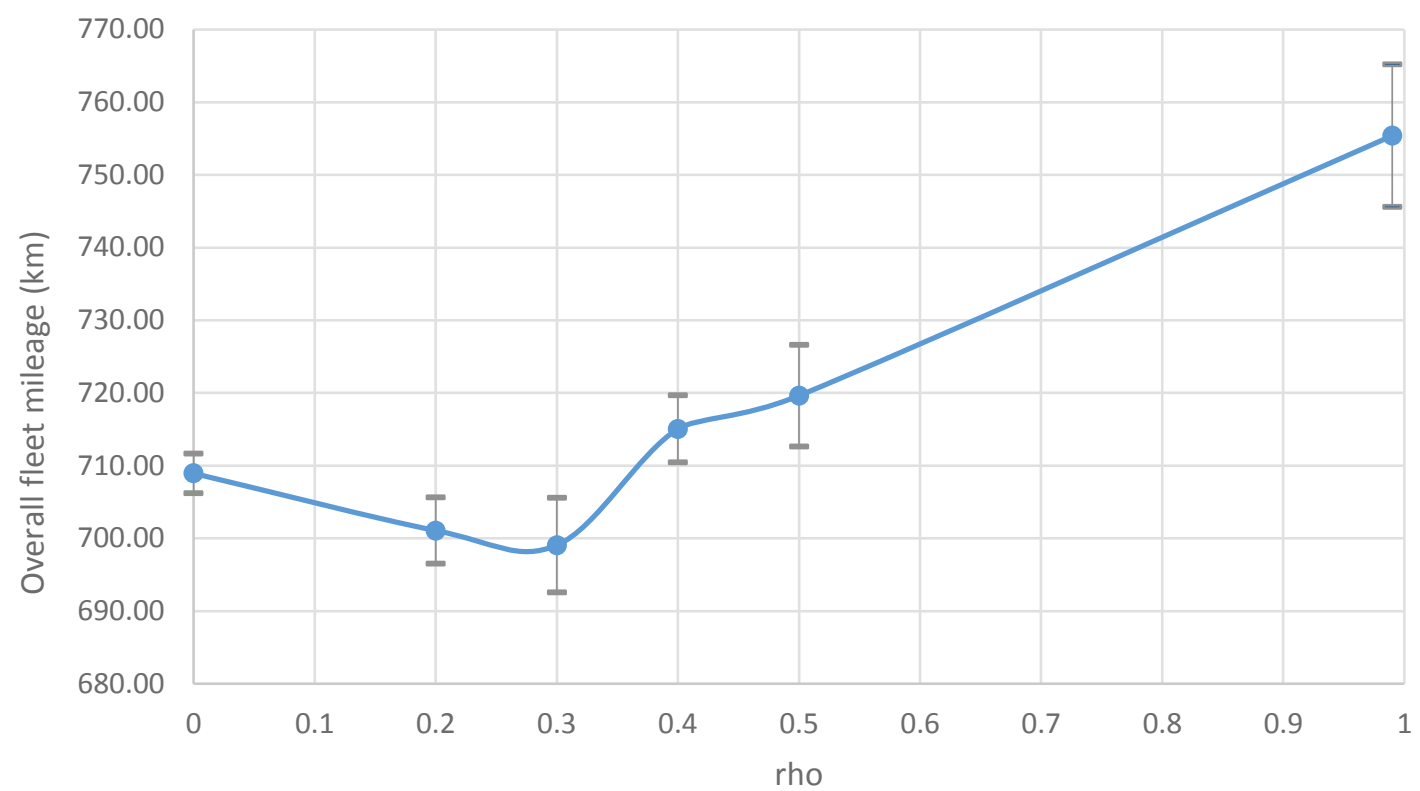

Fig. 4: The effect of pheromone evaporation factor (rho) on overall fleet mileage

\section{Beta $(\beta)$ and $Q 0$}

These two parameters are both related to the selection process of the next point to travel as explained in Section 3.2. The tuning process of these parameters are performed according to the literature and optimal values of $\beta=6$ and $Q_{0}=0.2$ are considered as discussed in previous studies (Gaertner \& Clark, 2005). The only difference here in the selection process of the next point comes from the limited range of EVs. In this study, an additional constraint is considered to check if the EV has enough charge to catch the next point and return to depot or not. If the charge is not enough (e.g. for the points at far distances), those points are automatically eliminated from the list of feasible next points.

Summarising all the parametric investigations for the ACO-based FMS algorithm, Table 4 gives the best values for each parameter that are used in this study.

Table 4: optimum values of the ACO parameters for FMS case-study

\begin{tabular}{cc}
\hline parameter & value \\
\hline number of ants & 50 \\
number of iterations & 500 \\
$\alpha$ (alpha) & 0.75 \\
$\rho$ (rho) & 0.3 \\
$\beta$ (beta) & 6 \\
$\mathrm{Q}_{0}$ & 0.2 \\
\hline
\end{tabular}




\section{FMS simulation using different initial conditions}

In this section, the effect of the initial condition on performance of the proposed ACObased FMS algorithm is investigated by repeating same simulation using different initial conditions as explained in Section 3.2. In Table 5, the overall fleet mileage is presented using different initialisation techniques for ACO FMS algorithm. The results demonstrate that the initial condition can significantly affect the overall FMS performance. In the first case presented in Table 5, random initial condition is considered for all EVs. In the second case, the ACO algorithm starts from the best initial point that is obtained by the nearestneighbour (NN) technique. In this case, the best initial condition is only obtained at the beginning for the first EV however, the rest of the fleet (other EVs) would start randomly. The use of NN initial condition for the first EV together with the optimum parameters of ACO algorithm, leads to the best solution. The application of the NN best initial condition means that the ACO algorithm starts from a very good solution at the beginning. Although, this is kind of restriction for ACO (comparing to a random initial condition), it helps the algorithm to generate better results because the restriction is not that much to freeze the performance of the algorithm. If the $\mathrm{NN}$ initial condition is applied repeatedly for all EVs (the third case in Table 5), the drawback is that the ACO algorithm becomes restricted too much in terms of exploring new solutions (in this case, the results of ACO become exactly the same to the nearest-neighbour technique). In other words, the advantage of the random initial condition is having more chance of getting the global optima as presented in Table 5.

Table 5: ACO best result using different initial conditions

\begin{tabular}{cc}
\hline Initial Condition & $\begin{array}{c}\text { Fleet overall } \\
\text { mileage }(\mathbf{k m}) \text { - best result }\end{array}$ \\
\hline Random initial condition for all EVs & 678.54 \\
NN initial condition for EV1 & 661.53 \\
NN initial condition for all EVs & 687.64 \\
\hline
\end{tabular}

\section{FMS simulation with various densities}

In this section, various densities of target points (introduced in Section 2.2) are investigated. The influence of density on performance of a FMS algorithm can be investigated in two aspects: (i) simulation time and (ii) total fleet mileage (objective 
function). For this purpose, similar simulations of ACO FMS algorithm are conducted for low, medium and high density cases (presented in Fig. 1). The results demonstrate significant effect of density on FMS algorithm's performance where the proposed algorithm performs quite well for low density. However, it might face more challenge when the density increases. In a simulation case as an example, the simulation time of 150 iterations is obtained 19 seconds for low density, 239 seconds for medium and 1925 seconds for the high density scenario. In terms of accuracy, ACO performs better at lower densities like other optimisation algorithms. More iterations are required at higher densities since the problem becomes more complex. In such case, a more powerful computer is needed to decrease the run time. In addition, proper initial condition and pheromone concentration parameter updating can also help to improve ACO FMS algorithm's performance at higher density scenarios. In all the following sections, 'medium' density is considered for consistency.

\section{Simulation results validation vs. Nearest Neighbour algorithm}

In this section, performance of the proposed FMS algorithm using ACO is validated against the Nearest-Neighbour (NN) algorithm. For this purpose, different versions of both algorithms are considered by changing the initial conditions and the adjustable parameters. As presented in Table 6, the proposed ACO FMS algorithm is able to decrease the overall fleet mileage, in the medium density case study, from $687.64 \mathrm{~km}$ (best NN performance) to $661.53 \mathrm{~km}$ (best ACO performance) that means $3.8 \%$ improvement. This means lots of energy saving when we look at it in larger scale. For example, in a fleet of twenty EVs where each EV has around $30 \mathrm{kWh}$ energy on board, the total daily energy consumption of the fleet is around $600 \mathrm{kWh}$. This is equivalent to $216,000 \mathrm{kWh}$ per year. So, $3.8 \%$ improvement in such a fleet would be equivalent to $8200 \mathrm{kWh}$ energy saving per year. This is a remarkable achievement since no additional investment is needed when applying such a change in FMS software.

Additionally ACO algorithm is able to explore and obtain a huge number of different solutions, whereas NN algorithm has a unique solution. ACO as a self-learning algorithm, although not proving the global minima, is able to examine and get a better solution no matter the randomness of the environment. Complementary results demonstrate that more improvement is achievable in the case of low density however, ACO's performance is 
not much better than $\mathrm{NN}$ in the case of high density. Consequently, ACO algorithm is suggested to be used up to a certain level of complexity and after that, simpler algorithms such as NN can have roughly same performance with less computational effort. Furthermore, the implementation of ACO in this research can be followed by future studies to try to make the algorithm closer to reality of applicable in a real case scenario. A clear advantage over NN which performance is always limited.

Fig. 5 shows performance of both NN and ACO FMS algorithms in a case-study when EVs are dispatched to cover certain number of target points at medium density. As shown in the figure, vehicles' routing is performed differently in the two cases. Although ACO performs better than $\mathrm{NN}$ in this case, there is no mathematical prove that the ACO gets the 'global optima'. So, there is a possibility to achieve even better results when using other algorithms.

Table 6: Nearest Neighbour and ACO FMS results comparison in medium density case-study

\begin{tabular}{ccc|c|c}
\hline \multicolumn{3}{c|}{ Nearest Neighbour (NN) } & \multicolumn{2}{c}{ ACO } \\
\hline $\begin{array}{c}\text { NN with } \\
\text { random } \\
\text { initial } \\
\text { condition }\end{array}$ & $\begin{array}{c}\text { NN with best } \\
\text { initial } \\
\text { condition for } \\
\text { EV1 }\end{array}$ & $\begin{array}{c}\text { NN with best } \\
\text { initial } \\
\text { conditions for } \\
\text { all EVs }\end{array}$ & $\begin{array}{c}\text { ACO with best } \\
\text { initial condition for } \\
\text { EV1 }\end{array}$ & $\begin{array}{c}\text { ACO with best initial } \\
\text { condition for EV1 and } \\
\text { optimum parameters }\end{array}$ \\
\hline 782.90 & 703.19 & 687.64 & 703.95 & 661.53 \\
\hline
\end{tabular}
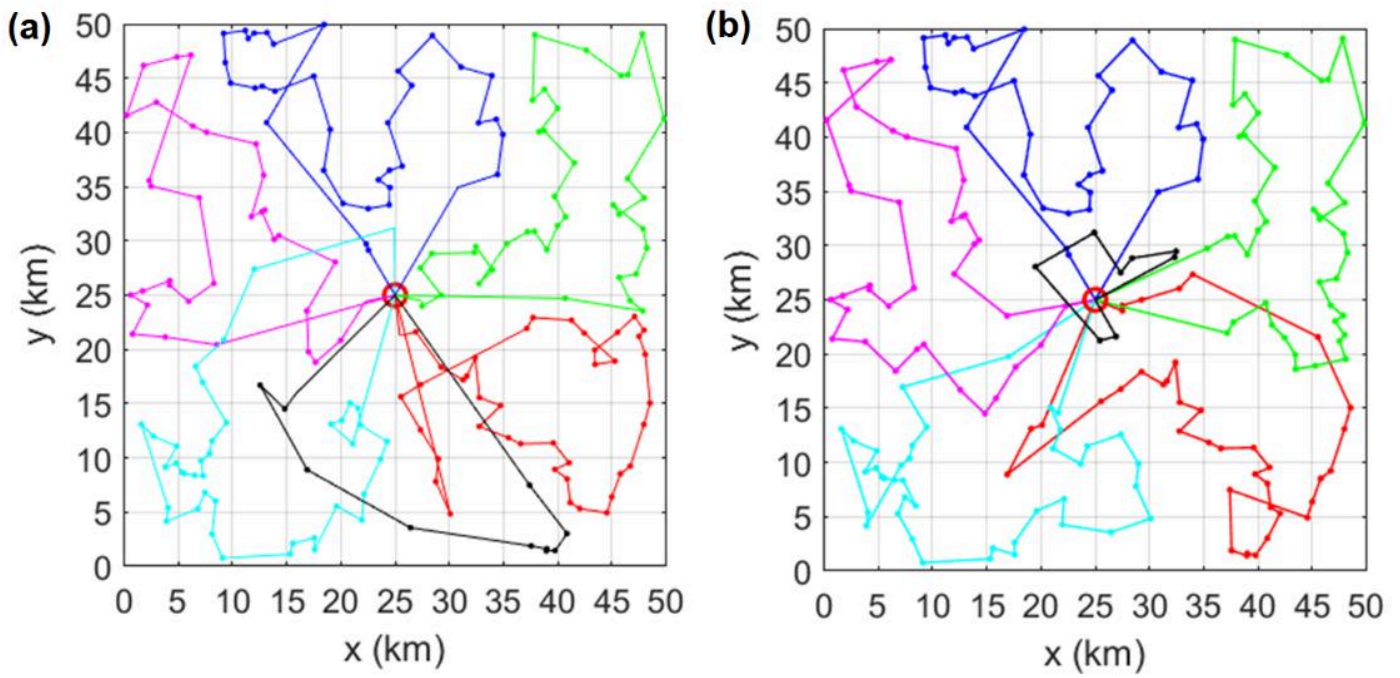

Fig. 5: FMS simulation in medium density case-study - each colour represents an EV's journey: (a) Nearest Neighbour, (b) ACO 


\section{Conclusions}

In this study, a new algorithm was developed based on ACO for fleet management and vehicle routing in an optimum way. Nearest-Neighbour algorithm was used as a benchmark to evaluate performance of the proposed algorithm. The results demonstrated that the new algorithm is able to improve fleet's performance $3.8 \%$ in terms of overall fleet mileage that can lead to a significant save of energy in large scale over time. Even more achievement is theoretically possible by using other versions of ACO or other algorithms since there is no mathematical prove that the proposed ACO algorithm gets the 'global optima'.

Pheromone concentration was found as the most important aspect to get ACO algorithm work properly and get the desired performance. Initial pheromone and pheromone updating together with evaporation, which involve $\alpha$ and $\rho$ parameters, are the main matters to accomplish the self-learning characteristic of the algorithm and make the best use of it for a FMS problem. In addition to the ACO parameters, the impacts of the initial condition and the level of density were investigated. The results demonstrated that initial condition has a significant effect on ACO FMS algorithm. According to the results, a combination of random and $\mathrm{NN}$ initial conditions can provide the best performance of the proposed ACO FMS algorithm. The influence of target points' density was also investigated by considering three levels of density. The main outcome was that the ACO algorithm's performance is restricted when complexity increases. In that case, a reasonably long time is required to do the optimisation. This cannot be a feasible solution particularly in an online application.

In this study, a particular FMS case-study was considered in which EV fleet is charged over night at depot to be ready for operation in the next day. In comparison with literature, ACO had been already used to perform the Travelling Salesman or Vehicle Routing problems. However, direct implementation on FMS is what gives novelty to the research. Combining both research areas is where real application can be done. Regarding recent battery technology developments and increasing range of EVs, e.g. Nissan LEAF 2018 with $40 \mathrm{kWh}$ battery capacity vs. the older version with $24 \mathrm{kWh}$, slow overnight charging can be a feasible solution if the range of EV would be enough for the whole day operation of the fleet. This would bring benefits like decreasing battery degradation due to fast 
charging, and also more efficient interaction between EV fleet and the grid. As an extension of this study, other scenarios can be considered as well. Examples are considering fast charging during the day or considering more than one depot. Additionally dynamic behaviour of the environment can be studied, e.g. the E-ACO done by $\mathrm{Xu}, \mathrm{Pu}$ and Duan (2018). The multi-depot case-study is categorised as a MTSP. In that case, more than optimising the position of the depot, which is usually given randomly, the objective is to show the distribution between depots and customers. A solutions of such a problem in the literature is Minimum-Maximum MTSP where the aim is to "minimise the maximum tour length of a single agent instead of minimising the overall sum of tour lengths by all agents" (Kivelevitch, Cohen, \& Kumar, 2012). The proposed FMS algorithm in this study is designed to work in offline condition however, an online version of it can be developed in future studies. In that case, the 'optimisation time' would be as important as the objective function that needs proper trade-offs.

\section{References}

Abousleiman, R., \& Rawashdeh, O. (2014). Energy-efficient routing for electric vehicles using metaheuristic optimization frameworks. 17th IEEE Mediterr. Electrotech. Conf., (pp. 298-304). Cernobbio, Italy.

Barco, J., Guerra, A., Muñoz, L., \& Quijano, N. (2017). Optimal Routing and Scheduling of Charge for Electric Vehicles: A Case Study. Mathematical Problems in Engineering, 1-16.

Bell, J. E., \& McMullen, P. R. (2004). Ant colony optimization techniques for the vehicle routing problem. Adv. Eng. Informatics, 18(1), 41-48.

Betz, J., Werner, D., \& Lienkamp, M. (2016). Fleet Disposition Modeling to Maximize Utilization of Battery Electric Vehicles in Companies with OnSite Energy Generation. Transp. Res. Procedia, 19, 241-257.

Boone, N., Sathyan, A., \& Cohen, K. (2015). Enhanced Approaches to Solving the Multiple Traveling Salesman Problem. AIAA Infotech @ Aerosp., 1-7.

Chao, Z. H., \& Xiaohong, C. (2013). Optimizing Battery Electric Bus Transit Vehicle Scheduling with Battery Exchanging: Model and Case Study. Procedia - Soc. Behav. Sci., 96, 2725-2736.

Chen, T. D., Kockelman, K. M., \& Hanna, J. P. (2016). Operations of a shared, autonomous, electric vehicle fleet: Implications of vehicle \& charging infrastructure decisions. Transp. Res. Part A, 94, 243-254. 
Chen, X., Kong, Y., Fang, X., \& Wu, Q. (2011). A fast two-stage ACO algorithm for robotic path planning . Neural Computing and Applications, 22(2), 313319.

Christofides, N. (1976). The vehicle routing problem. Revue Française a Automatique, Informatique et Recherche Opérationnel, 10(2), 55-70.

Dorigo, M., \& Gambardella, L. M. (1997). Ant colonies for the travelling salesman problem. Biosystems, 43(2), 73-81.

Erdoĝan, S., \& Miller-Hooks, E. (2012). A Green Vehicle Routing Problem. Transp. Res. Part E Logist. Transp. Rev., 48(1), 100-114.

Fotouhi, A., Auger, D. J., Cleaver, T., Shateri, N., Propp, K., \& Longo, S. (2016). Influence of Battery Capacity on Performance of an Electric Vehicle Fleet. 5th Int. Conf. Renew. Energy Res. Appl. Birmingham, UK.

Gaertner, D., \& Clark, K. (2005). On Optimal Parameters for Ant Colony Optimization algorithms TSP classifications. International Conference on Artificial Intelligence. Las Vegas, Nevada, USA.

Goel, R. and Maini, R. (2018) 'A hybrid of ant colony and firefly algorithms ( HAFA ) for solving vehicle routing problems', Journal of Computational Science, 25 Elsevier B.V., pp. 28-37.

Heris, S. M. (2015, September 4). MATLAB implementation of ACO for Discrete and Combinatorial Optimization Problems. Retrieved June 15, 2018, from https://uk.mathworks.com/matlabcentral/fileexchange/52859-ant-colonyoptimization-aco

Hu, J., Morais, H., Sousa, T., \& Lind, M. (2016). Electric vehicle fleet management in smart grids: A review of services, optimization and control aspects. Renewable and Sustainable Energy Reviews, 56, 1207-1226.

Jaradat, G. M. (2018). Hybrid elitist-ant system for a symmetric traveling salesman problem: case of Jordan. Neural Computing and Applications, 29(2), 565-578.

Johnson, S. (2001). Emergence: The Connected Lives of Ants, Brains, Cities and Software. Penguin Books.

Kivelevitch, E. H., Cohen, K., \& Kumar, M. (2012). A binary programming solution to the Multiple-Depot, Multiple Traveling Salesman Problem with constant profits. AIAA Infotech Aerosp. Conf. Exhib. Garden Grove, CA, USA.

Laporte, G. (1992). The traveling salesman problem: An overview of exact and approximate algorithms. Eur. J. Oper. Res., 59(2), 231-247.

Lin, S. (1965). Computer Solutions of the Traveling Salesman Problem. Bell Syst. Tech. J., 44(10), 2245-2269. 
Ma, J., Yang, T., Hou, Z.-G., Tan, M., \& Liu, D. (2007). Neurodynamic programming: a case study of the traveling salesman problem. Neural Computing and Applications, 17(4), 347-355.

Murakami, K. (2017) 'A new model and approach to electric and diesel-powered vehicle routing', Transportation Research Part E, 107 Elsevier Ltd, pp. 2337.

Narasimha, K. S., \& Kumar, M. (2011). Ant Colony Optimization Technique to Solve the Min-Max Single Depot Vehicle Routing Problem. American Control Conference, (pp. 3257-3262). San Francisco, CA, USA.

Ouaarab, A., Ahiod, B., \& Yang, X.-S. (2013). Discrete cuckoo search algorithm for the travelling salesman problem. Neural Computing and Applications, 24(7), 1659-1669.

Palmieri, N., Yang, X.-S., Rango, F. D., \& Marano, S. (2017). Comparison of bioinspired algorithms applied to the coordination of mobile robots considering the energy consumption. Neural Computing and Applications, 1-24.

Saji, Y., \& Riffi, M. E. (2015). A novel discrete bat algorithm for solving the travelling salesman problem. Neural Computing and Applications, 27(7), 1853-1866.

Schiffer, M., \& Walther, G. (2017). The electric location routing problem with time windows and partial recharging. Eur. J. Oper. Res., 260(3), 995-1013.

Tarasewich, P., \& McMullen, P. R. (2002). Swarm Intelligence: Power in Numbers. Commun. ACM, 45(8), 62-67.

Xu, H., Pu, P. and Duan, F. (2018) 'Dynamic Vehicle Routing Problems with Enhanced Ant Colony Optimization', 2018

Zhang, Q., \& Zhang, C. (2018). An improved ant colony optimization algorithm with strengthened pheromone updating mechanism for constraint satisfaction problem. Neural Computing and Applications, 30(10), 32093220.

Zhang, S., Gajpal, Y., Appadoo, S.S. and Abdulkader, M.M.S. (2018) 'Electric Vehicle Routing Problem with Recharging Stations for Minimizing Energy Consumption', International Journal of Production Economics, Elsevier B.V. 
2019-12-03

\section{Electric vehicle fleet management using ant colony optimisation}

Muriel, Javier Biera

IGI Global

Biera Muriel J, Fotouhi A. (2020) Electric vehicle fleet management using ant colony optimisation. International Journal of Strategic Engineering, Volume 3, Issue 1, 2020, Article number 1

https://doi.org/10.4018/lJoSE.2020010101

Downloaded from Cranfield Library Services E-Repository 